\title{
IS QCD AT SMALL X A STRING THEORY?
}

\author{
R. Peschanski \\ Service de Physique Théorique CEA-Saclay F-91191 Gif-sur-Yvette FRANCE
}

\begin{abstract}
Using the dipole picture describing the $1 / N_{C}$ limit of QCD at small $x$ and the conformal invariance properties of the BFKL kernel in transverse coordinate space, we show that the $1 \rightarrow p$ dipole densities can be expressed in terms of dual ShapiroVirasoro amplitudes $B_{2 p+2}$ and their generalization including non-zero conformal spins. We discuss the possibility of an effective closed string theory of interacting QCD dipoles.
\end{abstract}

\section{Introduction}

The QCD "hard Pomeron" is understood as the solution of perturbative QCD expansion at high energy $(W)$ after resumming the leading $\left(\alpha \ln W^{2}\right)^{n}$ terms. It is known to obey the BFKL equation 1 . It has recently attracted a lot of interest in relation with the experimental results obtained at HERA for deep-inelastic scattering reactions at very low value of $x \approx Q^{2} / W^{2}$, where $Q^{2}$ is the virtuality of the photon probe $\gamma^{*}$ and $W$ is, in this case, the c.o.m. energy of the $\gamma^{*}$-proton system. Interestingly enough, the proton structure functions increase with $W$ at fixed $Q$ in a way qualitatively compatible with the prediction of the BFKL equation 2 . However, the phenomenological discussion is still under way, since scattering of a "hard" probe on a proton is not a fully perturbative QCD process and, moreover, alternative explanations based on renormalization group evolution equations do exist 6 . On the other hand, the phenomenological success $B$ of parametrizations based on the BFKL evolution in the framework of the QCD dipole model $\mathrm{t}$ is quite encouraging for a further study of its properties.

Beyond these phenomenological motivations, there exist interesting related theoretical problems which we want to address in the present paper. In its 2dimensional version, the BFKL equation expresses 1 the leading-order resummation result for the elastic (off-mass-shell) gluon-gluon scattering amplitude in the $2-d$ transverse plane. Alternatively one introduces 5 the space coordinate variables via 2-dimensional Eourier transforms. Explicit solutions of the BFKL equation can be obtained 5 using conformal invariance properties of the BFKL kernel expressed in the 2-dimensional transverse coordinate space. Using these symmetry properties in the sequel, we will address the problem of finding the vertices for processes involving $2+2 p$ external gluon legs where $\rho_{0} \rho_{1}, \rho_{a_{0}} \rho_{a_{1}}, \ldots, \rho_{p_{0}} \rho_{p_{1}}$ are their arbitrary $2-d$ coordinates. Our main result is the explicit solution (cf. formula (5)) of the $p$-uple dipole density distri- 
butions in the transverse coordinate plane, and their expression, (cf. formula (8)) as integrands of dual Virasoro-Shapiro amplitudest. The expressions for arbitrary conformal spins (i.e. for all the conformal components) are found to be analoguous to generalizations of Shapiro-Virasoro amplitudes with excited states as external legs in a closed string theory.

\section{BFKL dynamics in the framework of the QCD dipole model}

Our starting point is the recently obtained result 6 that the $2+2$ amplitude is equal, up to kinematical factors, to the number density $n_{1}$ of dipoles appearing in the $1 / N_{C}$ QCD calculation of the wave-function of a massive quarkantiquark pair (onium) after an evolution "time" $Y$ (such can be interpreted the total available rapidity space $Y$ in the BFKL equation written as a diffusion process 9 ). In the QCD-dipole picture $\Theta$, gluons are equivalent to $q \bar{q}$ pairs (in the $N_{c} \rightarrow \infty$ limit) which recombine into a collection of independent and colorless dipoles. The elastic amplitude is thus obtained in the $N_{c} \rightarrow \infty$ limit from the elementary dipole-dipole amplitudes weighted by the dipole number densities of each initial state obtained after evolution "time" $Y$. Using conformal invariance properties of the BFKL kernel, it turns out 1 that:

$$
\begin{aligned}
n_{1}\left(\rho_{0} \rho_{1} ; \rho_{0}^{\prime} \rho_{1}^{\prime} \mid Y\right) & =\int d \omega e^{\omega Y} \sum_{n \in \mathcal{Z}} \int d \nu \frac{n_{1}^{n, \nu}\left(\rho_{0} \rho_{1} ; \rho_{0}^{\prime} \rho_{1}^{\prime}\right)}{\omega-\omega(n, \nu)} \\
& =\sum_{n \in \mathcal{Z}} \int d \nu e^{\omega(n, \nu) Y} n_{1}^{n, \nu}\left(\rho_{0} \rho_{1} ; \rho_{0}^{\prime} \rho_{1}^{\prime}\right),
\end{aligned}
$$

where $n_{1}^{n, \nu}$ are the components of the dipole density expanded upon the conformally invariant basis and

$$
\omega(n, \nu)=\frac{2 \alpha N_{c}}{\pi} \Re\left\{\psi(1)-\psi\left(\frac{1+n}{2}+i \nu\right)\right\}
$$

is the value of the BFKL kernel in the (diagonal) conformal basis. The corresponding eigenvectors are explicitly known $\mathrm{E}$ to be:

$$
E^{n, \nu}\left(\rho_{o \gamma}, \rho_{1 \gamma}\right)=(-1)^{n}\left(\frac{\rho_{0 \gamma} \rho_{1 \gamma}}{\rho_{01}}\right)^{\Delta_{\gamma}}\left(\frac{\bar{\rho}_{0 \gamma} \bar{\rho}_{1 \gamma}}{\bar{\rho}_{01}}\right)^{\widetilde{\Delta}_{\gamma}},
$$

with $\rho_{i j}=\rho_{i}-\rho_{j}$ (resp. $\left.\bar{\rho}_{i j}=\bar{\rho}_{i}-\bar{\rho}_{j}\right)$ are the holomorphic (resp. antiholomorphic) components in the 2- $d$ transverse plane represented by the complex plane $\mathcal{C}$ and $\Delta_{\gamma}=\frac{n-1}{2}-i \nu, \widetilde{\Delta}_{\gamma}=-\frac{n+1}{2}-i \nu,(n \in \mathcal{Z}, \nu \in \mathcal{R})$, are the quantum numbers defining the appropriate unitary representations 5 of the global conformal 
group $S L(2, \mathcal{C})$. Indeed the BFKL solution (i.e., also, the QCD dipole solution) is given by

$$
n_{1}^{n, \nu}\left(\rho_{0} \rho_{1} ; \rho_{0}^{\prime} \rho_{1}^{\prime}\right)=\frac{\nu^{2}+n^{2} / 4}{\left|\rho_{0^{\prime} 1^{\prime}}\right|^{2} \pi^{4} / 2} \int_{\mathcal{R}^{2}} d^{2} \rho_{\gamma} \bar{E}^{n, \nu}\left(\rho_{0 \gamma}^{\prime}, \rho_{1 \gamma}^{\prime}\right) E^{n, \nu}\left(\rho_{0 \gamma}, \rho_{1 \gamma}\right),
$$

which can be explicitly calculated in terms of hypergeometric functions 12 .

\section{Multiple-dipole vertices are dual string amplitudes}

In order to generalize these investigations to an arbitrary number of gluons, we shall use the QCD dipole formalism allowing to express the probability of finding $p$ dipoles in the wave-function of an initial one, i.e. the $p$-uple dipole density after an evolution $Y, n_{p}\left(\rho_{0} \rho_{1} ; \rho_{a_{0}} \rho_{a_{1}}, \rho_{b_{0}} \rho_{b_{1}}, \ldots, \rho_{p_{0}} \rho_{p_{1}} \mid Y\right) n_{p}$ is the solution of an integral equation which has been proposed in Ref. 13 , and approximate solutions have been worked out and applied to problems like the double and triple QCD Pomeron coupling 13 , dipole production 4 , hard diffraction 15 and, more generally, to the unitarization problem 13. In particular, Monte-Carlo simulations of the unitarization series based on a numerical resolution of the $n_{p}$ integral equations have been performed 16 .

However a general expression for the solution of these equations and a physical interpretation of its properties were still lacking. It is the purpose of our work to provide such a solution, which is intimately related, as we shall see, to dual string amplitudes emerging from the QCD dipole picture.

We now consider the p-uple distribution of dipoles $n_{p}^{n, \nu}$. One writes:

$$
\begin{aligned}
& n_{p}^{n, \nu}\left(\rho_{\gamma} ; \rho_{a_{0}} \rho_{a_{1}}, \ldots, \rho_{p_{0}} \rho_{p_{1}} \mid \omega\right)=\frac{1}{2 a(n, \nu)(\omega-\omega(n, \nu))} \frac{1}{\left|\rho_{a} \ldots \rho_{p}\right|} \times \\
& \sum_{n_{a}, \ldots, n_{p}} \int \frac{d \nu_{a} \ldots d \nu_{p}}{a\left(n_{a}, \nu_{a}\right) \ldots a\left(n_{p}, \nu_{p}\right)} \frac{1}{\omega\left(n_{a}, \nu_{a}\right)+\ldots+\omega\left(n_{p}, \nu_{p}\right)-\omega} \\
& \quad \int d^{2} \rho_{\alpha} \ldots d^{2} \rho_{\pi} \bar{E}^{n_{a}, \nu_{a}}\left(\rho_{a_{0} \alpha}, \rho_{a_{1} \alpha}\right) \ldots \bar{E}^{n_{p}, \nu_{p}}\left(\rho_{p_{0} \pi}, \rho_{p_{1} \pi}\right) \times \\
& \quad \int \frac{d^{2} \rho_{0} \ldots d^{2} \rho_{p}}{\left|\rho_{01} \rho_{12} \ldots \rho_{p 0}\right|^{2}} E^{n, \nu}\left(\rho_{0 \gamma}, \rho_{1 \gamma}\right) E^{n_{a}, \nu_{a}}\left(\rho_{1 \alpha}, \rho_{2 \alpha}\right) \ldots E^{n_{p}, \nu_{p}}\left(\rho_{p \pi}, \rho_{0 \pi}\right)(5)
\end{aligned}
$$

where we have used the fact that the probability kernel of finding $p$ dipoles at "time" $Y$ can be expressed by iteration of the single BFKL kernel, namely:

$$
\left|\frac{\rho_{01}}{\rho_{02} \rho_{12}}\right|^{2} \times\left|\frac{\rho_{02}}{\rho_{23} \ldots \rho_{(p-1) p} \rho_{p 0}}\right|^{2}=\left|\frac{\rho_{01}}{\rho_{12} \ldots \rho_{(p-1) p} \rho_{p 0}}\right|^{2} .
$$


Hence, the overall kernel is expressed in a symmetric way as a function of the coordinates of the perimeter designed by the coordinates $\rho_{0}, \rho_{1} \ldots \rho_{p-1}, \rho_{p}$ independently of the possible intermediate steps (cf. the $\rho_{0} \rho_{2}$ segment).

Let us consider the last term, i.e. the vertex part $V_{\gamma \ldots \pi}$, of formula (5). Inserting the definitions (3) in the expression of $V_{\gamma \ldots \pi}$, one gets:

$V_{\gamma \ldots \pi}=\int_{\mathcal{C}{ }^{p+1}} \frac{d^{2} \rho_{0} \ldots d^{2} \rho_{p}}{\left|\rho_{01} \ldots \rho_{p 0}\right|^{2}} \times\left(\frac{\rho_{0 \gamma} \rho_{1 \gamma}}{\rho_{01}}\right)^{\Delta_{\gamma}} \ldots\left(\frac{\rho_{p \pi} \rho_{0 \pi}}{\rho_{p 0}}\right)^{\Delta_{\pi}} \times\{\rho \Rightarrow \bar{\rho}, \Delta \Rightarrow \tilde{\Delta}\}$.

Our observation is that $V_{\gamma \ldots \pi}$ can be expressed as the integrand of a Shapiro-

Virasoro amplitude $B_{2 p+2}$. One may write

$$
\begin{array}{r}
B_{2 p+2}=\int_{\mathcal{C}^{p+1}} \frac{d^{2} \rho_{\alpha} \ldots d^{2} \rho_{\pi}}{\mathcal{V}_{3}} \prod_{\eta_{i}<\eta_{j}}^{p+1} \rho_{\eta_{i} \eta_{j}}^{-\Delta_{\eta_{i} \eta_{j}}} \bar{\rho}_{\eta_{i} \eta_{j}}^{-\tilde{\Delta}_{\eta_{i} \eta_{j}}} V_{\gamma \ldots \pi}= \\
=\int_{\mathcal{C}^{2+2 p}} \frac{d^{2} \rho_{\alpha} \ldots d^{2} \rho_{\pi}}{\mathcal{V}_{3}} d^{2} \rho_{0} \ldots d^{2} \rho_{p}\left(\rho_{0 \gamma} \rho_{1 \gamma}\right)^{\Delta_{\gamma}}\left(\rho_{1 \alpha} \rho_{2 \alpha}\right)^{\Delta_{\alpha}} \ldots\left(\rho_{p 1 \pi} \rho_{p \pi}\right)^{\Delta_{\pi}} \\
\times \prod_{\eta_{i}<\eta_{j}}^{p+1} \rho_{\eta_{i} \eta_{j}}^{-\Delta_{\eta_{i} \eta_{j}}}\left(\rho_{01}\right)^{-1-\Delta_{\gamma}} \ldots\left(\rho_{p 0}\right)^{-1-\Delta_{\pi}} \times\{\rho \Rightarrow \bar{\rho}, \Delta \Rightarrow \tilde{\Delta}\},
\end{array}
$$

where the conformal dimensions $\Delta_{\eta_{i} \eta_{j}},\left(\eta_{i}, \eta_{j} \in(\alpha \ldots \pi)\right)$, are fixed by global $S L(2, \mathcal{C})$ invariance $17, \mathbb{E}$. The factor $\mathcal{V}_{3}^{-1}$ formally corresponds to the "division" by the volume of the group $S L(2, \mathcal{C})$. In practice 18 one may arbitrarily fix three coordinates $\rho_{\eta_{1}}, \rho_{\eta_{2}}, \rho_{\eta_{3}}$, and write

$$
\mathcal{V}_{3} \equiv \frac{d^{2} \rho_{\eta_{1}} d^{2} \rho_{\eta_{2}} d^{2} \rho_{\eta_{3}}}{\left|\rho_{\eta_{1}} \rho_{\eta_{2}} \rho_{\eta_{3}}\right|^{2}}
$$

Eqn.(14) is a particular realization of the Shapiro Virasorg amplitudes obtained as bosonic string tree amplitudes. Indeed, by definition 6 , one writes:

$$
B_{N}=\int \prod_{j=1}^{N} \frac{d^{2} \rho_{j}}{\mathcal{V}_{3}} \prod_{i<j}^{N} \rho_{i j}^{-p_{i j}} \bar{\rho}_{i j}^{-\tilde{p}_{i j}}
$$

where the powers $p_{i j}$ and $\tilde{p}_{i j}$ obey a set of $S L(2, \mathcal{C})$ constraints 6 . These constraints are automatically satisfied in our case, since the global invariance is respected at every step. The powers $p_{i j}$ appearing in (8) are known combinations of conformal dimensions $\Delta$ and $\tilde{\Delta}$ corresponding to particular realizations of the $S L(2, \mathcal{C})$ constraints. 


\section{A closed string theory for QCD at low $\mathrm{x}$ ?}

The Shapiro-Virasoro amplitudes which are obtained for $(1 \rightarrow p)$ dipole distributions lead naturally to the question of a closed-string interpretation of the high-energy limit of perturbative QCD. Indeed, these amplitudes appear in the context of a closed string moving in a Minkowskian $(1, d-1)$ target space 1 . More generally, such amplitudes appear as a consequence of vertex operator constructions in conformal field theories 19 and are related to the existence of an (anomalous) infinite-dimensional algebra associated with local conformal invariance, namely the Virasoro algebra. Moreover in the case of a critical target-space dimension $d_{c}$, the Fock space on which the quantum string theory is realized is spanned by positive normed states (no ghosts) with full reparametrization invariance. This connection has both a practical and conceptual interest for QCD calculations. First, the many and much explored mathematical properties of dual amplitudes may lead to a simplification of QCD dipole computations for given processes, e.g. "hard" diffraction, multiPo! ! meron contributions, etc.. Second, there is a possibility of building an effective theory of QCD in the high-enrgy limit, which could be based on a string theory (instead of a field theory). This would allow the computation of string loop contributions and thus induce an effective theory of interacting QCD Pomerons.

However, the variables which appear as conformal exponents $p_{i j}$ of the integrands are not directly expressed as scalar products of momenta in a Minkowskian $(1, d-1)$ target space. They are complex numbers obeying constraints which are not directly expressed as on-mass shell and momentum conservation constraints as for the closed string 6 . Even if such a target-space interpretation is possible, an analytic continuation in the imaginary direction (implied by the quantum numbers of the conformal eigenvectors (3)) is to be performed. It is thus useful to pass in review the properties of Shapiro-Virasoro amplitudes in this context and to see which are those to be completed for a full closed string theory to be valid.

\section{i) Duality}

A first consequence of the solutions (5),(8) is that duality properties exist in the dipole formulation of QCD vertices. Indeed, by construction, ShapiroVirasoro amplitudes are meromorphic and forbid the existence of multiple pole singularities coming from dual channels. For instance, the $\left(\rho_{0}, \rho_{2}\right)$ and $\left(\rho_{1}, \rho_{i}\right)$, $i=3 \ldots p$ channels appearing in (5), (6) cannot bring simultaneous singularities to the amplitude. As usual in dual theories, there exists intricate relations between different ways of describing the amplitudes depending on the series of pole contributions which are choosen for their expansion. An interesting first 
example of such a duality property has been indeed provided by the equivalence of the "t-channel" BFKL elastic 4-gluon amplitude with the "s-channel" QCD dipole description of the same amplitude6. Further applications of this fruitful concept are expected from our results.

ii) Non-zero conformal spins

As a practical consequence of our identification of the multiple-dipole vertices with integrands of standard Shapiro-Virasgro amplitudes in the case of zero conformal spins, one may use some tools 20 which are developed in the string theoretical formalism to generalize our investigations to the case of general (integer or half-integer) conformal spins as follows; One can consider in general amplitudes of the form:

$$
B_{N}=\int \prod_{i=1}^{N} d \rho_{i}\left[\frac{d \rho_{\alpha} d \rho_{\beta} d \rho_{\delta}}{\left|\rho_{\alpha \beta} \rho_{\beta \delta} \rho_{\delta \alpha}\right|^{2}}\right]^{-1} \prod_{i<j}^{N} \rho_{i j}^{-p_{i j}+\frac{n_{i j}}{2}} \bar{\rho}_{i j}^{-p_{i j}+\frac{\tilde{n}_{i j}}{2}}
$$

where $n_{i j}, \tilde{n}_{i j}$ are integers. Interestingly enough, in the framework of string theory, this corresponds to consider external excited states of the bosonic string. Moreover, the same techniques allow to connect closed string to open string tree amplitudes which may allow to extend to the multiple-vertex calculations the conformal-block structure initially identified in the BFKL 4-point amplitudes 6 .

\section{iii) Extended conformal symmetry and Virasoro algebra}

In the original paper of Ref. $\mathrm{O}$, it has been noticed that it was not straightforward to extend the (already beautiful) global conformal symmetry $S L(2, \mathcal{C})$ to the infinitely dimensional conformal group in 2 dimensions. In other words, only the 6 generators of the Virasoro algebra: $\mathcal{L}_{-1}, \mathcal{L}_{1}, \mathcal{L}_{0}$ (3 holomorphic) and their 3 non-holomorphic counterparts, were expected to generate the symmetry algebra of the BFKL kernel. The results we obtain indicate that the algebra can probably be extended to the infinite series of locally conformal generators $\mathcal{L}_{n}, n \in \mathcal{Z}$, i.e. the whole Virasoro algebra, at least in the QCD dipole representation. as usual, the symmetry is expected to be anomalous due to the possibility of a central charge 19 (conformal anomaly) at the quantum level of consistency. This issue will depend on the interpretation of a suitable $p$-independent target-space representation of the exponents $p_{i j}$. For instance, if an embedding $p_{i j} \rightarrow p_{i} \cdot p_{j}$ in a Minkowskian $(1, d-1)$ space is allowed, this will determine the central charge to be related to $d$ and the critical dimension to be $d_{c}=26$, by compensation of the ghost contribution due to reparametrization symmetry 6 . This interesting issue certainly deserves more study. 
Acknowledgments

We want to thank Andrzej Bialas and Henri Navelet for a fruitful collaboration on the QCD theory of dipoles which initiated the present work. Christophe Royon and Samuel Wallon are acknowledged for stimulating discussions.

\section{References}

1. L.N.Lipatov, Sov. J. Nucl. Phys. 23 (1976) 642; V.S.Fadin, E.A.Kuraev and L.N.Lipatov, Phys. lett. B60 (1975) 50; E.A.Kuraev, L.N.Lipatov and V.S.Fadin, Sov.Phys.JETP 44 (1976) 45, 45 (1977) 199; I.I.Balitsky and L.N.Lipatov, Sov.J.Nucl.Phys. 28 (1978) 822

2. A general review of the experimental and phenomenological situation with opening views on the future can be found in: Future Physics at HERA, Proceedings of the Workshop 1995/96 (G.Ingelman, A.De Roeck, R.Klanner Eds., http://www.desy.de/ heraws96).

3. H.Navelet, R.Peschanski, Ch.Royon and S.Wallon Phys. Lett. $\mathbf{B 3 8 5}$ (1996) 357.

4. A.H.Mueller, Nucl. Phys. B415 (1994) 373. (See also in a different framework: N.N.Nikolaev and B.G.Zakharov, Zeit. fur. Phys. $\mathbf{C 4 9}$ (1991) 607; ibid. C64 (1994) 651).

5. L.N.Lipatov Zh. Eksp. Teor. Fiz.90 (1986) 1536 (Sov. Phys. JETP 63 (1986) 904).

6. H.Navelet and R.Peschanski, Conformal Invariance and the exact solution of BFKL equations, Saclay preprint t97/016, hep-ph/9703238, to be published in Nucl. Phys.B.

7. M.A.Virasoro, Phys. Rev. 177 (1969) 2309; J.A.Shapiro, Phys. lett. B33 (1970) 361.

8. P.H.Frampton, Dual Resonance Models , (1974, Benjamin; New edition: 1986, World Scientific).

9. See, e.g. J.Bartels, A.De Roeck, M.Loewe, Z.Phys. C 54 (1992) 635.

10. Z.Chen, A.H.Mueller, Nucl. Phys. B451 (1995) 579.

11. J.Bartels, Zeit. fur. Phys. C60 (1993) 471; Phys. lett. B298 (1993) 204; J.Bartels, M.Wüsthoff Zeit. fur. Phys. C66 (1995) 157; J.Bartels, L.N.Lipatov, M.Wüsthoff Nucl. Phys. B464 (1996) 298. For a review, see H.Lotter, PHD thesis, hep-ph/9705288 and references therein; I thank H. Lotter, G. Korchemsky and Al Mueller for fruitful correspondence and discussions on this point.

12. L.N.Lipatov, Small-x physics in perturbative QCD, hep-ph/9610276.

13. A.H.Mueller, Nucl. Phys. B437 (1995) 107. See also: A.H.Mueller and B.Patel, Nucl. Phys. B425 (1994) 471.

14. A. Bialas and R. Peschanski, Phys. lett. B355 (1995) 301. 
15. A. Bialas and R. Peschanski, Phys. lett. B378 (1996) 302; B387 (1996) 405.

16. G. P.Salam, Nucl. Phys. B461 (1996) 512; A. H.Mueller and G. P.Salam, Nucl. Phys. B475 (1996) 293.

17. A.M.Polyakov, Zh. Eksp. Teor. Fiz. Lett.12 (1970) 538, 66 (1974) 23.

18. Z.Koba and H.B.Nielsen, Nucl. Phys. B10 (1969) 633, B12 (1969) 517.

19. For a recent book on conformal field theories and useful references, see Ph.Di Francesco, P.Mathieu, D. Senechal, Conformal Field theory, (1997, Springer-Verlag).

20. H.Kawai, D.C.Lewellen and S.-H.H.Tye, Nucl. Phys. B464 (1996) 298. See also, for a related method: Vl.S. Dotsenko and V.A. Fateev, Nucl. Phys. B240 FS12 (1984) 312; 